\title{
$h$-Adaptive Finite Element Solution of Unsteady Thermally Driven Cavity Problem
}

\author{
David A. Mayne, Asif S. Usmani and Martin Crapper \\ School of Civil and Environmental Engineering, University of Edinburgh
}

\begin{abstract}
An $h$-adaptive finite element code for solving coupled Navier-Stokes and energy equations is used to solve the thermally driven cavity problem for Rayleigh numbers at which no steady state exists (greater than $1.9 \times 10^{8}$ ). This problem is characterised by sharp thermal and flow boundary layers and highly advection dominated transport, which normally requires special algorithms, such as streamline upwinding, to achieve stable and smooth solutions. It will be shown that $h$-adaptivity provides a suitable solution to both of these problems (sharp gradients and advection dominated transport). Adaptivity is also very effective in resolving the flow physics, characterised by unsteady internal waves and separation zones. Fundamental frequencies, generated by unsteady internal waves, are calculated for three Rayleigh numbers; $2 \times 10^{8}, 3 \times 10^{8}$ and $4 \times 10^{8}$ using a Prandtl number of 0.71 and results are compared with other published results.
\end{abstract}

\section{Introduction}

It is well known that $h$-adaptive FEM is very well suited to modelling scalar and vector fields containing sharp gradients by automatically refining the spatial discretisation to 'fit' the solution. The refinement is normally based on some a-posteriori estimation of the discretisation error. In previous papers $[1,2]$ the authors have clearly shown that for transient flow and transport problems, where advection is the dominant mechanism, $h$-adaptive FEM fulfils another very important role. It removes the requirement of introducing any special algorithm for treatment of the 'wiggles' generated by using numerical schemes which are essentially of a 'central difference' type, as is the case with the standard Galerkin finite element formulation, often referred to as GFEM. There has been a great deal of controversy over the special schemes that are used to 'suppress the wiggles' [3], however some of the best schemes, for instance SUPG [4], have been highly successful in providing a mathematically consistent framework, by using non-Galerkin formulations for such problems. In a previous paper [1], Usmani clearly demonstrated that if $h$-adaptive 
FEM is used for transient pure-advection problem (the rotating cone or cosine-hill problem) than the GFEM and SUPG solutions are practically indistinguishable. This was a confirmation of the original assertion by Gresho and Lee [3], 'don't suppress the wiggles they are telling you something'. The authors tested this further [2] for a coupled flow and heat transfer problem (thermally driven cavity problem for Rayleigh numbers up to $10 \times 10^{8}$ ) with the same conclusion. The exercise here is partly to test the $h$-adaptive GFEM solution procedure further, for even higher Rayleigh numbers when no steady state solutions exist.

Modelling the effects of a temperature difference across a square cavity has many important technical applications. A thorough understanding of the convective processes present at high Rayleigh numbers is critical in assessing the transport of heat in nuclear reactors, solar collectors and buildings. The thermally driven cavity problem also serves as a convenient benchmark test for new programs [5], which is another purpose of this exercise, as the authors are using this program (CADTRAS) to model the transport of cohesive sediments in estuarine waters, which are characterised by sharp density interfaces. The program was thoroughly tested by solving the thermally driven cavity problem for Rayleigh numbers up to $1.0 \times 10^{8}$ [2] and comparing results in considerable detail with the best available benchmark solutions. In this paper detailed solution of the same problem is undertaken for Rayleigh numbers; $2 \times 10^{8}, 3 \times 10^{8}$ and $4 \times 10^{8}$.

Bergholz [6] and Patterson et al [7] both discuss important features that are present in the development of a transient solution for high Rayleigh number cavity flows. Prandtl number strongly influences the transient development of the buoyancy driven flow features. The separation and recirculation observed in the departing corners becomes less pronounced and eventually disappears as the Rayleigh number is increased $[6,8]$. The corner regions are particularly important in the development of the flow over time. Ivey [9] proposed that the corner flow regions were characteristic of a hydraulic jump however Ravi et al [8] have concluded that this was not possible for several reasons. Among these

- Theory of hydraulic jumps does not explain the separation of flow at the horizontal boundaries.

- There is no substantial energy loss associated with the departing corner flow.

- The Froude number dependency appears to be arbitrary.

They propose that the flow structure in the departing corner is solely dependent on thermal effects, producing a separation and recirculation of the boundary layer. They also state that the separation zone that characterises the departing corner for high Rayleigh number flows, does not form beyond a Prandtl number of 1.2, similarly the recirculation zone disappears for Prandtl numbers above 1.4. They go on to say that this is due to the core temperature distribution suppressing large undershoots of temperature at the boundaries. Several researchers discuss the oscillatory behaviour of the flow at high Rayleigh number due to internal wave instability, [10-13]. Chenoweth and Paolucci [10] present power spectra plots of temperature time trace data, giving values of two key frequencies 
that dominate high Rayleigh number flows; the frequency of the boundary layer on the vertical wall and the frequency of wave breaking at the departing corners. The decrease in thickness of the boundary layer with increasing Rayleigh number imposes a constraint on the solution of the problem, $[10,12,14]$, requiring a high level of discretisation.

\section{Governing equations}

The governing equations have been written for a constant density, incompressible Newtonian fluid using the Boussinesq approximation to model buoyancy.

Continuity

$$
\nabla \cdot \mathbf{v}=0 \text { on } \Omega
$$

where $\mathbf{v}$ represents the velocity and $\Omega$ represents the domain.

Navier-Stokes

$$
\rho\left(\frac{\partial \mathbf{v}}{\partial t}+\mathbf{v} \cdot \nabla \mathbf{v}\right)+\nabla P=\nabla \cdot \mu\left[\nabla \mathbf{v}+(\nabla \mathbf{v})^{T}\right]-\rho \mathbf{g} \beta\left(T-T_{r}\right) \quad \text { on } \quad \Omega
$$

subject to boundary conditions:

$$
\begin{gathered}
\mathbf{F}=P \mathbf{n}-\mu\left[\nabla \mathbf{v}+(\nabla \mathbf{v})^{T}\right] \cdot \mathbf{n} \text { on } \Gamma_{F} \\
\mathbf{v}=\overline{\mathbf{v}}(x, y, t) \quad \text { on } \Gamma_{v}
\end{gathered}
$$

and initial conditions:

$$
\mathbf{v}(t=0)=\mathbf{v}_{\mathbf{o}} \quad \text { with } \quad \nabla \cdot \mathbf{v}_{\mathbf{o}}=0
$$

$\mu$ is the dynamic viscosity, $\mathbf{g}$ is the acceleration due to gravity, $\beta$ is the volumetric coefficient of thermal expansion, $T$ is the temperature, $T_{r}$ is a reference temperature, $\mathbf{F}$ represents the applied tractions on the boundary $\Gamma_{F}, n$ is the unit normal vector and $\overline{\mathbf{v}}$ is the Dirichlet boundary condition for velocity on the part of the boundary $\Gamma_{v}$.

Energy

$$
\frac{\partial T}{\partial t}+\mathbf{v} \cdot \nabla T=\nabla \cdot \kappa \nabla T \text { on } \Omega
$$

subject to boundary conditions:

$$
\mathbf{n} \cdot(\kappa \nabla T)=q \text { on } \Gamma_{Q}
$$




$$
T=\bar{T}(x, y, t) \quad \text { on } \quad \Gamma_{T}
$$

and initial conditions:

$$
T(t=0)=T_{o}
$$

where $q$ is a specified normal heat flux on the boundary $\Gamma_{Q}, \bar{T}$ is the Dirichlet boundary condition for temperature on the boundary $\Gamma_{T}$ and $\kappa$ is the thermal diffusivity given by,

$$
\kappa=\frac{k}{\rho C_{p}}
$$

where, $k$ is the thermal conductivity, $\rho$ is the fluid density and $C_{p}$ is the specific heat capacity.

\subsection{Finite Element Formulation}

The program is based on the Galerkin Finite Element Method (GFEM), solving for the primitive variables: $u$-velocity, v-velocity and T-temperature at all nodes in the mesh and P-pressure at a reduced level of interpolation to avoid spurious pressure modes, using a mixed formulation for the Navier-Stokes equations. The Navier-Stokes and energy equations were coupled by the Boussinesq approximation for buoyancy. Notation used here is as used by Gresho et al, $[15,16]$. The Galerkin FEM discretisation produces a system of ODE's as follows:

Navier-Stokes

$$
\left[\begin{array}{ccc}
\mathbf{M}_{\mathbf{u}} & 0 & 0 \\
0 & 0 & 0 \\
0 & 0 & \mathbf{M}_{\mathbf{v}}
\end{array}\right]\left(\begin{array}{c}
\dot{\mathbf{u}} \\
\dot{\mathbf{P}} \\
\dot{\mathbf{v}}
\end{array}\right)+\left[\begin{array}{ccc}
\mathbf{K}_{\mathbf{u u}} & \mathbf{C}_{\mathbf{u}} & \mathbf{K}_{\mathbf{u v}} \\
\mathbf{C}_{\mathbf{u}}^{\mathbf{T}} & 0 & \mathbf{C}_{\mathbf{v}}^{\mathbf{T}} \\
\mathbf{K}_{\mathbf{v u}} & \mathbf{C}_{\mathbf{v}} & \mathbf{K}_{\mathbf{v v}}
\end{array}\right]\left(\begin{array}{c}
\mathbf{u} \\
\mathbf{P} \\
\mathbf{v}
\end{array}\right)=\left(\begin{array}{c}
\mathbf{F}_{\mathbf{u}} \\
0 \\
\mathbf{F}_{\mathbf{v}}
\end{array}\right)
$$

Where $\mathbf{M}, \mathbf{K}, \mathbf{C}$ and $\mathbf{F}$ represent the mass matrix, viscous stress matrix, pressure gradient matrix and global force vector respectively. The first to third rows represent the $x$-momentum, continuity and $y$-momentum equation respectively. The right hand side vector $\mathbf{F}_{v}$ contains the coupling buoyancy term.

\section{Energy}

$$
\left[\mathbf{M}_{\mathbf{T}}\right](\dot{\mathbf{T}})+\left[\mathbf{K}_{\mathbf{T}}\right](\mathbf{T})=\left(\mathbf{F}_{\mathbf{T}}\right)
$$

Expansion of all terms can be found in Usmani et al [17]. The two systems of equations above are solved as a coupled system, with the $\mathbf{K}_{\mathbf{T}}$ term containing the velocities (obtained from solving the flow field) and the $\mathbf{F}_{\mathbf{v}}$ term containing the buoyancy forces (determined by the temperature field). 


\subsection{Temporal discretisation}

Temporal discretisation of the time domain is achieved by applying the generalised midpoint rule, $[18,19]$.

$$
\left[\frac{\mathbf{M}_{\mathbf{n}+\alpha}}{\alpha \Delta t}+\mathbf{K}_{\mathbf{n}+\alpha}\right]\left(\theta_{n+1}\right)=\left[\frac{\mathbf{M}_{\mathbf{n}+\alpha}}{\alpha \Delta t}-\frac{(1-\alpha)}{\alpha} \mathbf{K}_{\mathbf{n}+\alpha}\right]\left(\theta_{n}\right)+\frac{\left(\mathbf{F}_{\mathbf{n}+\alpha}\right)}{\alpha}
$$

Variation of $\alpha$ leads to different members of this family of methods i.e.

$\alpha=0$-Forward Difference or Forward Euler.

$\alpha=\frac{1}{2}$-Midpoint rule or Crank Nicolson.

$\alpha=\frac{2}{3}$-Galerkin.

$\alpha=1$-Backward Difference or Backward Euler.

The Crank Nicolson, Galerkin and Backward Euler schemes are all unconditionally stable, however, of these methods the oscillation limit is lowest for $\alpha=\frac{1}{2}$. The time step size chosen for all Rayleigh numbers is small enough to avoid an oscillatory solution when using $\alpha=\frac{1}{2}$. The choice of unconditionally stable implicit methods is enforced by the use of $h$-adaptivity as the smallest elements determine the stability of conditionally stable explicit methods, which makes them impractical for use in this context.

The formulations described above were implemented in the implicit transient FE code CADTRAS (Coupled Advective Diffusive TRAnSport model), which was used to solve the thermally driven cavity problem. The code incorporates an unstructured Delaunay triangulation based mesh generator [20], which allows automatic adaptive re-meshing to take place at each time step if necessitated by the a-posteriori error estimation algorithm. Six-node triangular elements are used for all the meshes.

\section{Adaptivity}

The use of $h$-adaptivity enables the solution of this problem at high Rayleigh number without the necessity of designing a suitable mesh at first and going through a trial-and-error process. Adaptivity automatically produces an optimal mesh based on a user specified discretisation error thus saving computational time and focusing effort intelligently over successive time steps on areas of high scalar gradients (which for this problem coincide with the areas of high velocity gradients). There are five distinct steps to the iterative adaptive process used here :

1. Solution of the coupled system

2. Recovery of smoothed scalar gradients using the super-convergent patch recovery (SPR) method [21] 
3. Error Estimation using the a-posteriori error calculated at all nodes in the mesh for the scalar field

4. Re-meshing based on the mesh sizes produced from the previous step

5. Transfer of all data to the new mesh

\section{Recovery}

The temperature field generated by the finite element method is most accurate at nodal points whereas the temperature gradients are most accurate at Gaussian integration points, known as the super-convergence phenomenon. Hinton and Campbell [22] showed that finite elements produce superior values of temperature gradient at node points after application of a smoothing procedure. Their method was based on a global smoothing scheme requiring the solution of a large system of equations. A more efficient and effective procedure was introduced by Zienkiewicz and Zhu [21], called super-convergent patch recovery (SPR). The smoothed nodal gradients are calculated from the Gauss points on a patch of elements surrounding a node, using a least squares interpolation, for each node in the mesh.

\section{Error Estimation}

The error estimator used was originally derived for heat conduction [23]. Mathematical justification of using such an estimator for the problem of this paper does not exist, however as the estimator used is based on the scalar flux, it has proven very effective in detecting regions of high scalar gradient, which in practice is sufficient for the purposes of this paper. The a-posteriori error is based upon an energy norm (see [23]),

$$
\|e\|^{2}=\int_{\Omega}(\nabla T)^{T} \kappa \nabla T d \Omega-\int_{\Omega}(\nabla \hat{T})^{T} \kappa \nabla \hat{T} d \Omega
$$

if we define,

$$
\begin{aligned}
& \|Q\|^{2}=\int_{\Omega}(\nabla T)^{T} \kappa \nabla T d \Omega \\
& \|\hat{Q}\|^{2}=\int_{\Omega}(\nabla \hat{T})^{T} \kappa \nabla \hat{T} d \Omega
\end{aligned}
$$

then Equation (12) can be rewritten as

$$
\|e\|^{2}=\|Q\|^{2}-\|\hat{Q}\|^{2}
$$

Such a definition allows a practical representation of the error norm in terms of a percentage error $\eta$,

$$
\eta=\frac{\|e\|}{\|Q\|} \mathrm{x} 100 \%
$$

Re-meshing and mesh generation

Specification of a permissible error $\bar{\eta}$ determines the level of refinement throughout the mesh, leading to a predicted reduction or increase in the element sizes so that the new 
mesh may possess an approximately equal distribution of error. The maximum permissible error for each element is calculated as,

$$
\|\hat{e}\|_{e}=\bar{\eta}\left(\frac{\|Q\|^{2}}{m}\right)^{\frac{1}{2}}
$$

where $m$ is the number of elements, $\bar{\eta}$ is the specified maximum percentage error. Dividing $\|\hat{e}\|_{e}$ by the calculated error in an element yields a parameter $\xi_{e}$ as follows,

$$
\xi_{e}=\frac{\|e\|_{e}}{\|\hat{e}\|_{e}}
$$

i.e. if $\xi_{e}>1$ the mesh must be refined in the vicinity of element $e$, conversely, if $\xi_{e}<1$ the mesh may be coarsened. The new element size is calculated using,

$$
\overline{h_{e}}=\frac{h_{e}}{\xi_{e}^{\frac{1}{p}}}
$$

where $h_{e}$ is the original element size and $p$ is the order of the element shape functions.

\section{Mesh data transfer}

Ensuring proper transfer of variables between meshes is crucial for conservation of quantities such as energy and momentum. A transfer strategy using local coordinates of nodal points and elemental shape functions has been used that maps the mesh data accurately. The local coordinates $(\xi-\eta)$ of each node in the adapted mesh are determined with respect to the elements of the previous mesh. Element shape functions are then used to interpolate the data onto the new mesh nodes.

\section{The Benchmark Problem}

The problem involves modelling fluid flow in a two dimensional square cavity of typical dimension $L$ with the two vertical walls being maintained at a temperature difference of $\Delta T$. The top and bottom walls are insulated and the velocities at all boundaries set to zero. The fluid inside the cavity is initially at rest and at a temperature which is the mean of the temperatures on the vertical walls. The resulting flow can be described by the Rayleigh number:

$$
\mathrm{Ra}=\mathrm{GrPr}=g \beta \frac{\Delta T L^{3}}{\nu \kappa}
$$

where $\mathrm{g}$ is the acceleration due to gravity, $\beta$ is the coefficient of volumetric expansion, $\mathrm{L}$ the typical dimension of the cavity, $\Delta T$ is the temperature difference between the vertical walls, $\nu$ is the kinematic viscosity and $\kappa$ is the thermal diffusivity. 
The following non-dimensional groups are used in the analysis and presentation of the computational results:

Velocity

$$
\begin{aligned}
& u^{*}=\frac{u L}{\kappa} \\
& v^{*}=\frac{v L}{\kappa}
\end{aligned}
$$

Temperature

$$
T^{*}=\frac{T-T_{2}}{T_{1}-T_{2}}
$$

Coordinates

$$
\begin{aligned}
x^{*} & =\frac{x}{L} \\
y^{*} & =\frac{y}{L}
\end{aligned}
$$

Time

$$
t^{*}=\frac{\kappa t}{L^{2}}
$$

where ${ }^{*}$ indicates the the non-dimensional quantity. $T_{1}$ and $T_{2}$ are the fixed temperatures at the two side walls of the cavity.

The Nusselt number is calculated at each node in the domain using

$$
\mathrm{Nu}=u T-\frac{\partial T}{\partial x}
$$

where the temperature gradient is obtained by the gradient recovery process.

\subsection{Departing Corner Flow}

It is important to understand the mechanism that generates the destabilising internal waves, dictating the pattern of the flow field. As mentioned in the introduction, Ravi et al [8] set out a description of the flow behaviour in the departing corners and give a mechanism for its creation. The left cavity region next to the vertical boundary carries flow at large velocities. This flow, after departing the corner, slows down, the isotherms that were packed closely together at the wall boundary spread out over a much thicker layer. The highest velocity layer, nearest the to the hot boundary experiences the greatest change in velocity after passing the departing corner. A slightly cooler layer (travelling at a slightly lower velocity) next to the hot layer is forced to slide over it in the corner region. This causes a sharp reversal in velocity as the cooler boundary layer plunges abruptly back into the cooler core, resulting in the characteristic u-shape isotherm. At high Rayleigh numbers the downward force of the negatively buoyant plume is enough to cause separation of flow from the horizontal boundary. Recirculation occurs when the fluid is re-entrained into the vertical wall boundary from the plume. 


\section{Results}

Values of u-velocity, v-velocity and temperature were recorded over the duration of the simulation for all three Rayleigh numbers; $2 \times 10^{8}, 3 \times 10^{8}$ and $4 \times 10^{8}$. They where recorded at a point $x=0.1032, y=0.8036$ within the unit square cavity, following Chenoweth et al [10]. This point falls in a particularly sensitive location regarding the oscillatory nature of the boundary layer. Figures 1 and 2 show time trace histories for all three variables. The temperature time history data was also converted from the time domain into the frequency domain using Fast Fourier Transform (FFT) analysis, this allows frequencies that characterise the time plots to be seen more clearly, see Figure 2.

The graphs showing primitive variable time histories for $\mathrm{Ra}=2 \times 10^{8}$, figures $1(\mathrm{a}), 1(\mathrm{~b})$, 2(a) show convergence to a periodic oscillation. Each plot is dominated by one fundamental frequency. This fundamental frequency is generated by the internal boundary layer instability at the departing corners.

Figure 2(b) shows one very clear spike, indicating the fundamental frequency, with a value of $546.9 \mathrm{~Hz}$. The $\mathrm{Ra}=3 \times 10^{8}$ time histories show a clear waveform consisting of more than one frequency, exhibiting quasi-periodic behaviour. The FFT plot, figure $2(\mathrm{~d})$, reveals a clear fundamental frequency at $651.0 \mathrm{~Hz}$. followed by several small, high frequency components. The time history graphs for $\mathrm{Ra}=4 \times 10^{8}$ show mildly chaotic, quasi-periodic behaviour, as previously shown by Chenoweth and Paolucci [10]. The fundamental frequency as per figure $2(\mathrm{f})$ is $781.3 \mathrm{~Hz}$. There is also an increased amount of high frequency background noise.

It is clear from the results that an increase in Rayleigh number is accompanied by an increase in the fundamental frequency of the oscillation. Chenoweth et al [10] present a table of results showing a similar increase in frequency with Rayleigh number however the values they obtained were slightly higher; $630.3,737.7$ and 850.2 for $\mathrm{Ra}=2 \times 10^{8}$, $3 \times 10^{8}$ and $4 \times 10^{8}$ respectively.

The frequency plot for $\mathrm{Ra}=3 \times 10^{8}$, figure $2(\mathrm{~d})$, shows a clear high amplitude fundamental frequency followed by several low amplitude high frequencies. The amplitude of the fundamental frequency is significantly larger than that of $2 \times 10^{8}$ and $4 \times 10^{8}$. For $3 \times 10^{8}$ the majority of the spectral energy resides in this spike while in the other Rayleigh numbers this energy is divided up between the fundamental frequency and other more substantial higher frequency components. The spectral plots presented by Chenoweth and Paolucci [10] show the same phenomenon but on a log scale for amplitude.

\subsection{Rayleigh number behaviour scale}

Figure 3 has been constructed on the basis of results presented in several research papers, $[10,12,24]$. The first important threshold marked on the diagram is $\mathrm{Ra}=1.9 \times 10^{8}$. This represents the transition from steady state flow to unsteady periodic flow, as recorded 


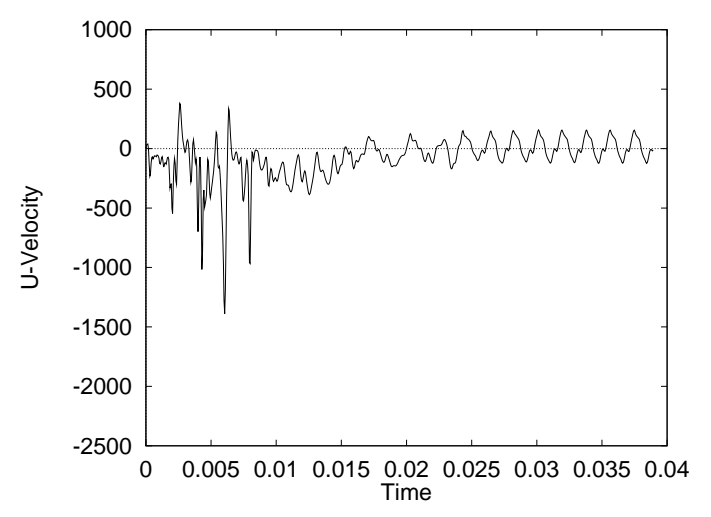

(a) U-Velocity, $\mathrm{Ra}=2 \times 10^{8}$

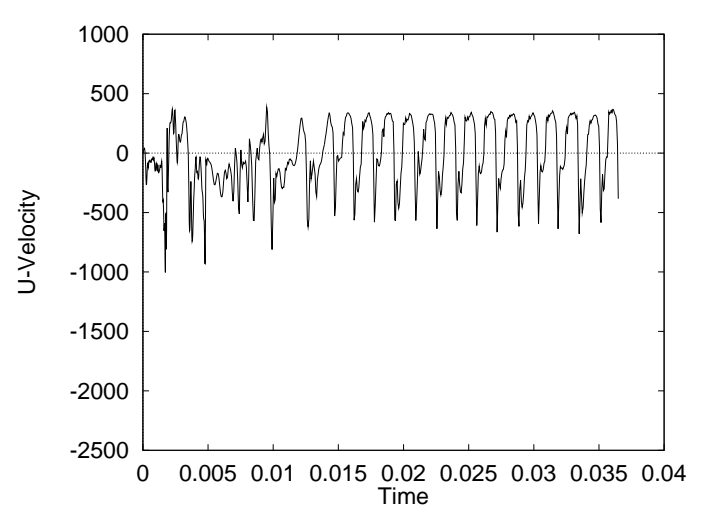

(c) U-Velocity, Ra $=3 \times 10^{8}$

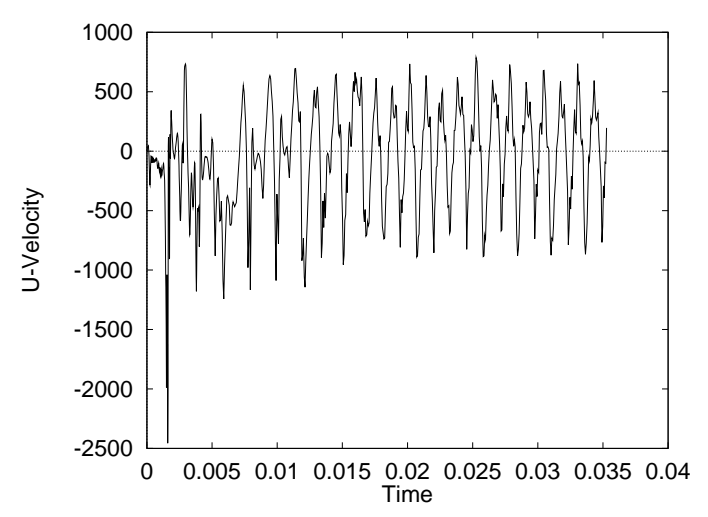

(e) U-Velocity, Ra $=4 \times 10^{8}$

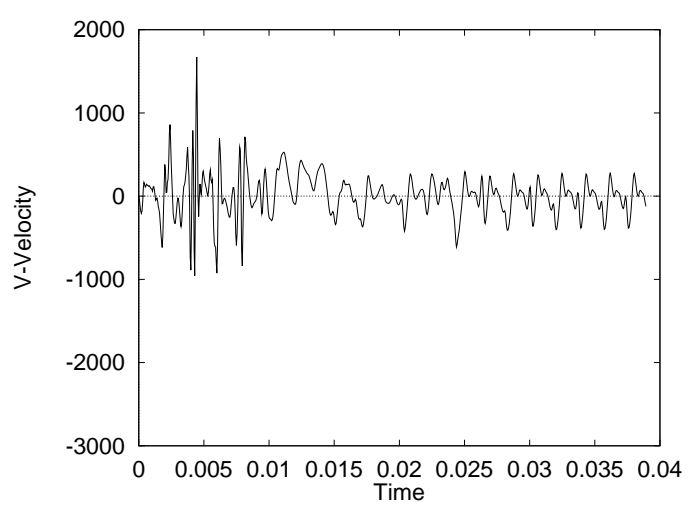

(b) V-Velocity, Ra $=2 \times 10^{8}$

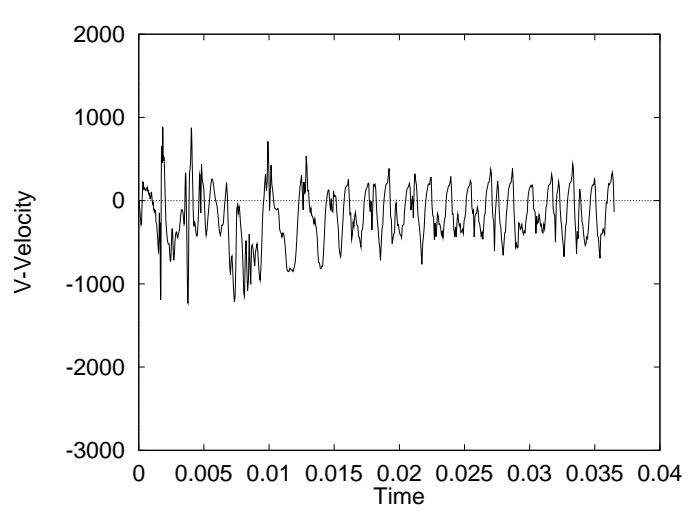

(d) V-Velocity, Ra $=3 \times 10^{8}$

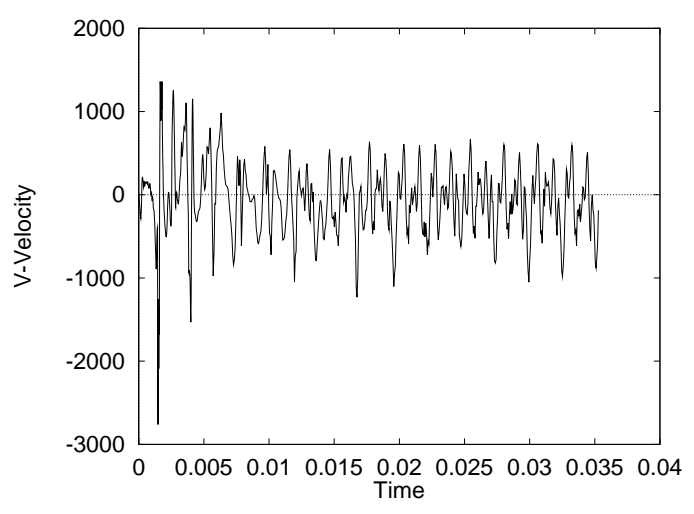

(f) V-Velocity, Ra $=4 \times 10^{8}$

Figure 1: Time trace histories at $\mathrm{x}=0.1032, \mathrm{y}=0.8036$ for $\mathrm{U}$ and $\mathrm{V}$ velocities. 


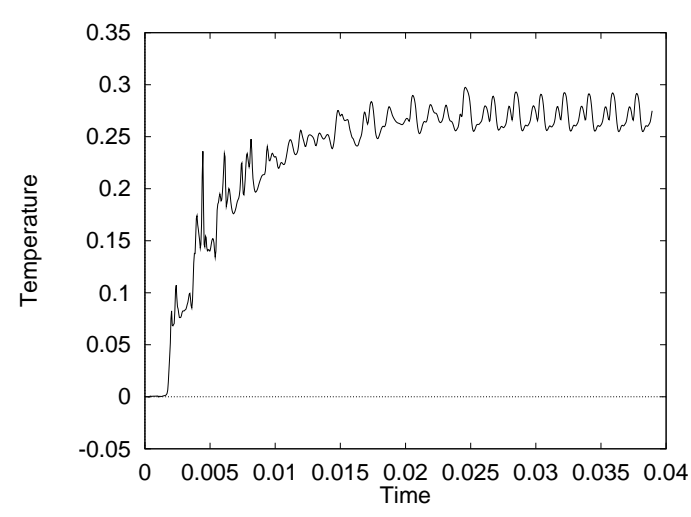

(a) Temperature, $\mathrm{Ra}=2 \times 10^{8}$

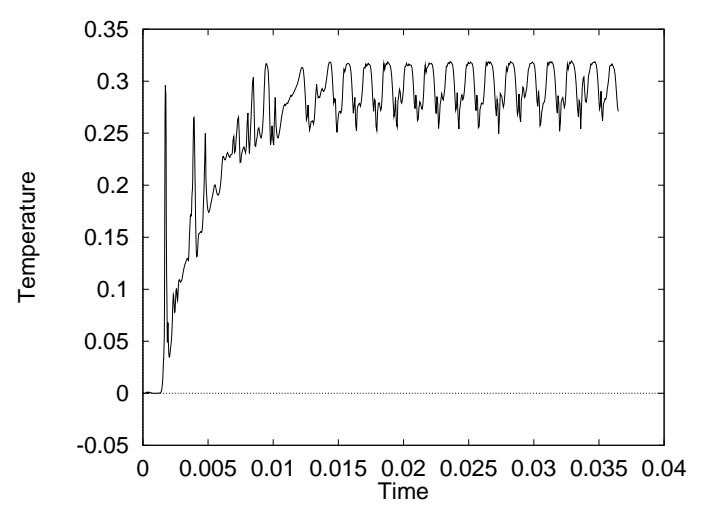

(c) Temperature, Ra $=3 \times 10^{8}$

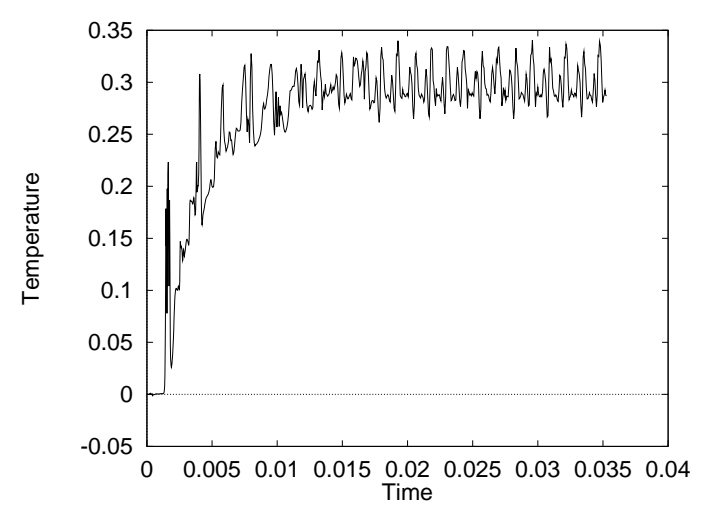

(e) Temperature, $\mathrm{Ra}=4 \times 10^{8}$

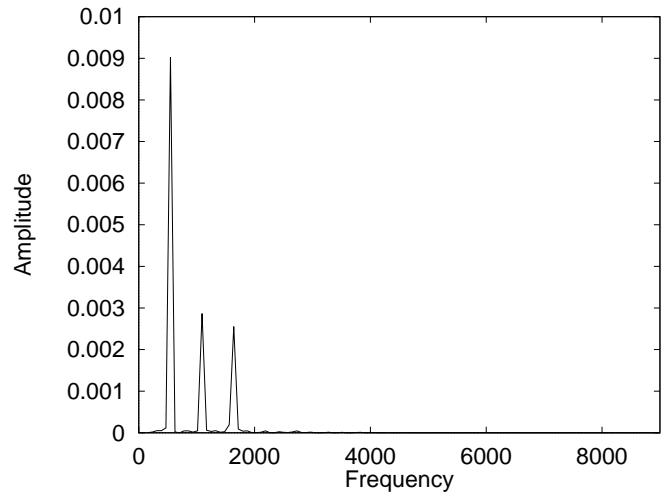

(b) FFT, Ra $=2 \times 10^{8}$

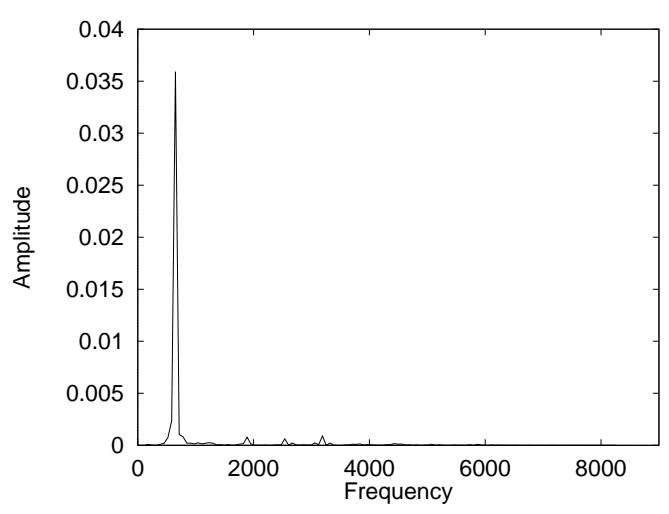

(d) FFT, Ra $=3 \times 10^{8}$

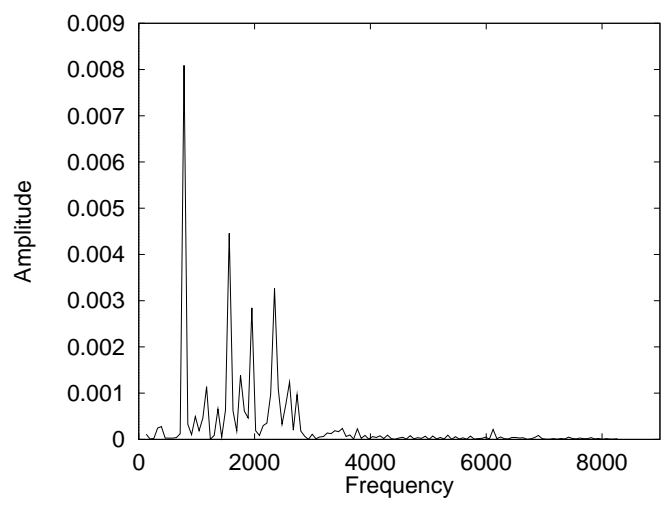

(f) $\mathrm{FFT}, \mathrm{Ra}=4 \times 10^{8}$

Figure 2: Time trace histories and FFT plots at $\mathrm{x}=0.1032, \mathrm{y}=0.8036$ for temperature 


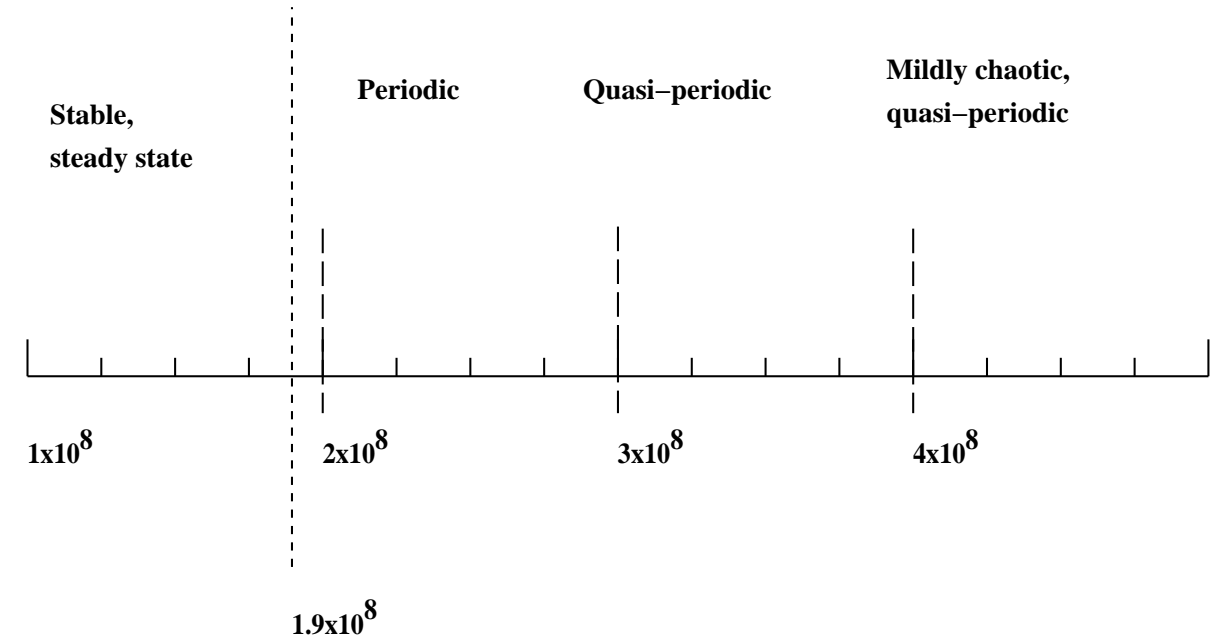

Figure 3: Rayleigh number versus behaviour

by Chenoweth et al [10] and Le Quere [25]. Chenoweth and Paolucci go on to predict two more regions of transition; instability of the wall boundary layers leading to quasiperiodic flow near $2.7 \times 10^{8}$ and a further change to mildly chaotic flow somewhere between $3 \times 10^{8}$ and $4 \times 10^{8}$. Very similar behaviour is noticed in the presented results, in that at $\mathrm{Ra}=2 \times 10^{8}$ the flow is periodic, at $3 \times 10^{8}$ the flow is clearly quasi-periodic and at $4 \times 10^{8}$ the flow is still maintains its quasi-periodic nature but shows signs of chaoticity, see Chenoweth et al figure 13 [10].

\section{$5.2 h$-adaptivity and its role in the solution}

Figure 4 shows a sequence of meshes produced during the solution of the thermally driven cavity problem for $\mathrm{Ra}=4 \times 10^{8}$. There were a total of 750 time steps producing 13 separate adaptive meshes during the simulation, six are shown to highlight the effective capture of important flow features. The corresponding velocity vectors and temperature contours are shown in Figures 5 and 6.

Mesh 1 - This is the pre-adaptive mesh i.e. the result of a number of re-meshing cycles based on the boundary conditions. The area around the vertical boundary layers is heavily discretised to capture the steep temperature gradients.

Mesh 6 - The boundary layer has rounded the corner and is moving across the horizontal surface. The mesh follows the temperature front as it moves, some degree of flow separation is manifested in the mesh at the departing corner.

Mesh 8 - The boundary layer is half way across the cavity, there are two distinct regions that form the leading edge of the intrusion; the separated zone and the boundary layer still attached to the horizontal surface.

Mesh 10 - The boundary layer has reached the opposite vertical boundary. A continuous plume stretches across the cavity. 


\begin{tabular}{|c|c|c|c|}
\hline & $2 \times 10^{8}$ & $3 \times 10^{8}$ & $4 \times 10^{8}$ \\
\hline \hline Numax, $y$ & $6.7140 \times 10^{-3}$ & $6.7031 \times 10^{-3}$ & $6.7328 \times 10^{-3}$ \\
\hline Numin, $y$ & 1.0 & 1.0 & 0.9938 \\
\hline
\end{tabular}

Table 1: Nusselt number positions for each Rayleigh number

Mesh 12 - The boundary layer has diffused into its surroundings to some degree causing the temperature gradients to decrease. The mesh has coarsened in these areas accordingly.

Mesh 13 - The highest level of discretisation is focussed in the departing corners capturing the zone of boundary layer recirculation. The centre of the recirculating eddy is just visible as an area of lower discretisation near the corner. The flow has settled down considerably, however the separated boundary layer is moving back and forth quasi-periodically. This is the last re-meshing cycle of the run, the temperature gradients are only varying around the departing corners and they have been discretised adequately to capture the unsteady internal waves.

Figures 5 to 7 show the development of the velocity field and temperature contours over time. The isotherms become increasingly stratified resulting in the distribution shown in Figure $7(\mathrm{f})$. The flow is mildly chaotic and unsteady but still retains a high degree of structure. The asymmetry of the flow, apparent in the isotherms and velocity vector plots in figures 5, 6 and 7, is mentioned by Chenoweth and Paolucci [10]. They suggest that the loss of symmetry is due to the quasi-periodic nature of the flow generated by presence of two different fundamental frequencies i.e. the internal wave and wall boundary oscillations.

Figure 8 shows the time history of temperature recorded at two points for $\mathrm{Ra}=2 \times$ $10^{8}$, one at $x=0.1032, y=0.8036$ and the other at $x=0.8968, y=0.1964$. The fundamental frequencies of the two time traces are very similar but there is an obvious difference between the two time history plots in figure 8. Unfortunately the lower time trace seems 'damped' compared to the top trace, this is due to the effect of unstructured mesh generation. The application of a structured mesh generator should remove this problem and allow a thorough analysis of any possible phase differences, however this is beyond the scope of this paper.

Table 1 shows the vertical positions of of maximum and minimum Nusselt numbers for the three Rayleigh numbers presented. Figures 9, 10 and 11 show the variation of Nusselt number over a period of time. The maximum, minimum and average Nusselt number on the boundary $x=0.0$ plotted against dimensionless time are shown. All nine plots show that the value of Nusselt number at the vertical boundary show periodic variation. The time histories of maximum and average Nusselt numbers show small amplitude oscillation while the minimum Nusselt number is more sensitive to the unsteady nature of the flow, exhibiting larger amplitude oscillation, becoming more pronounced with increasing Rayleigh number. 


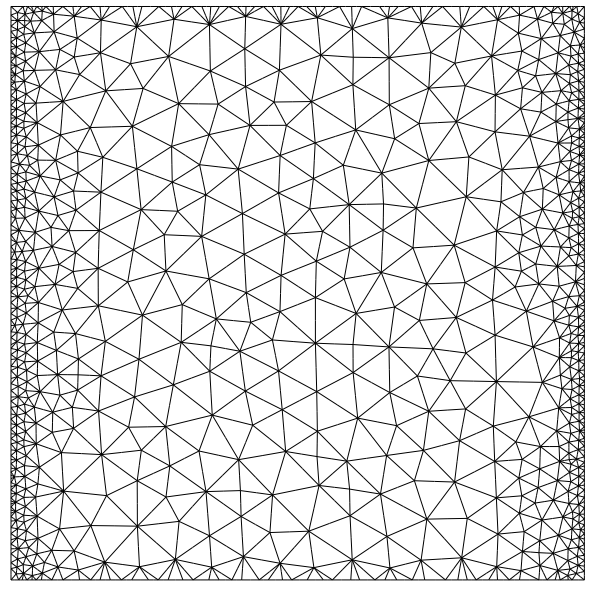

(a) Time step 1, mesh 1

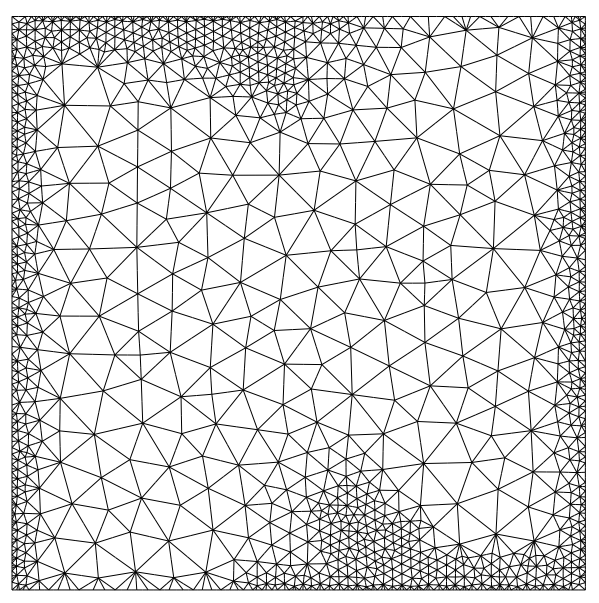

(c) Time step 22, mesh 8 .

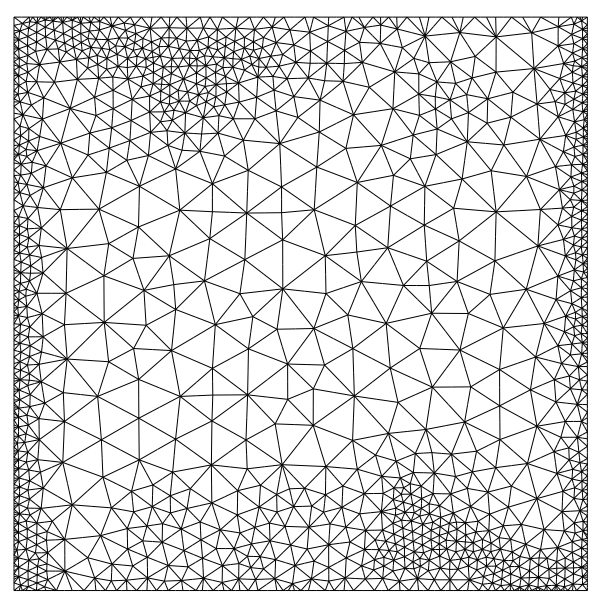

(e) Time step 69, mesh 12 .

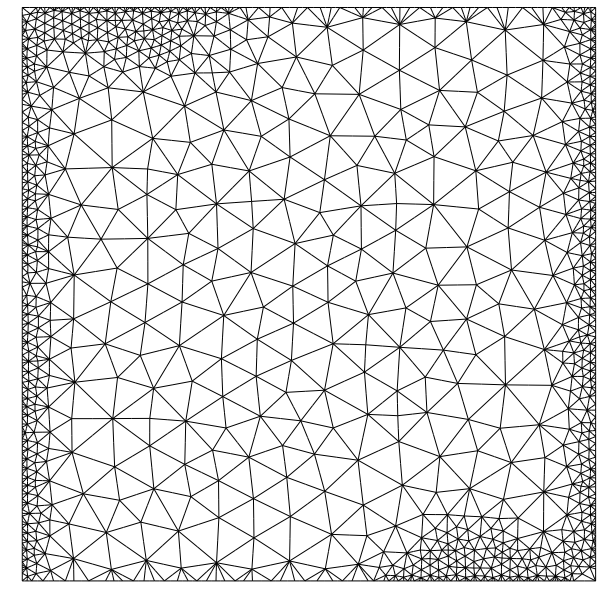

(b) Time step 14, mesh 6 .

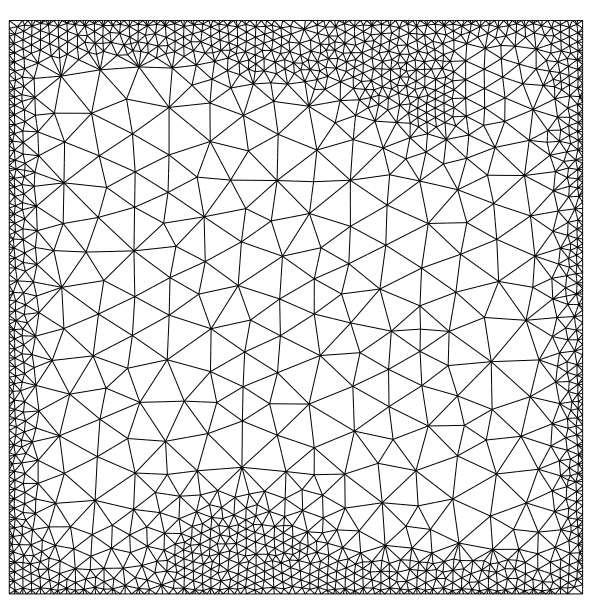

(d) Time step 33, mesh 10.

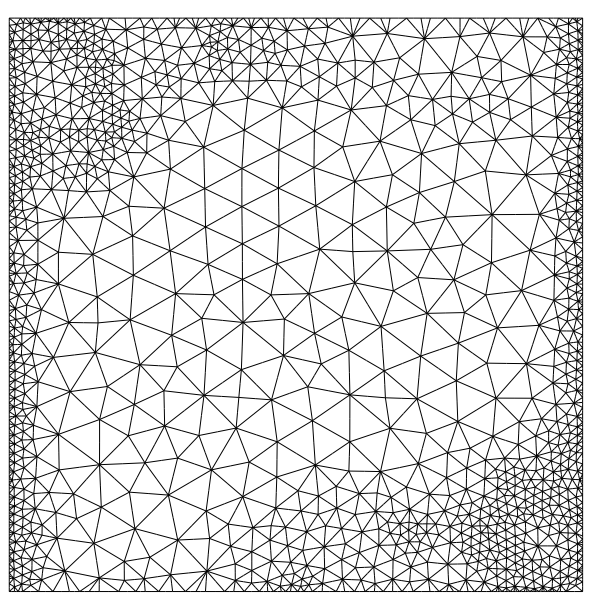

(f) Time step 78, mesh 13.

Figure 4: Adaptive mesh files for $\mathrm{Ra}=4 \times 10^{8}$ at (a) $t^{*}=0.0$; (b) $t^{*}=0.00028$; (c) $t^{*}=0.00044 ;$ (d) $t^{*}=0.00066 ;$ (e) $t^{*}=0.00138$; (f) $t^{*}=0.00156$. 


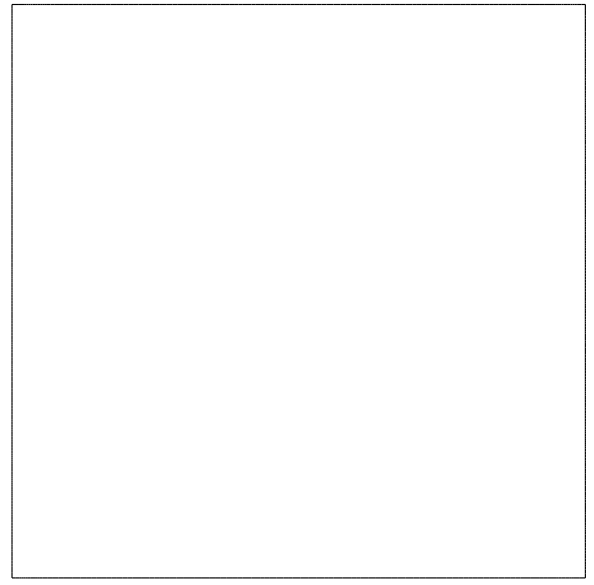

(a) Time step 1, velocity vectors.

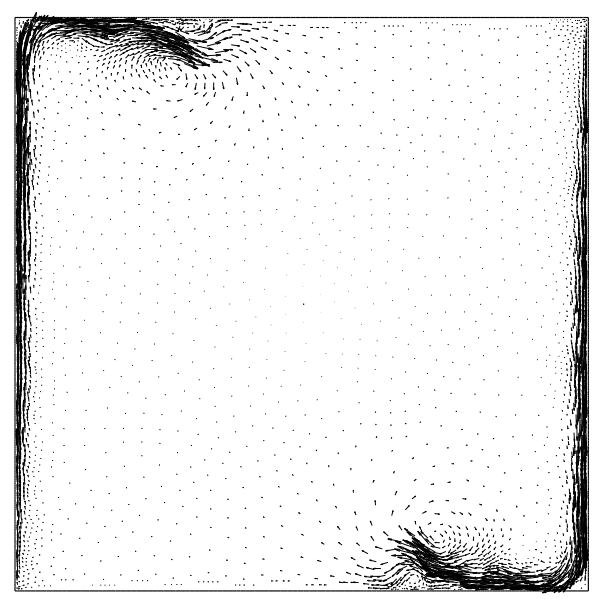

(c) Time step 14, velocity vectors.

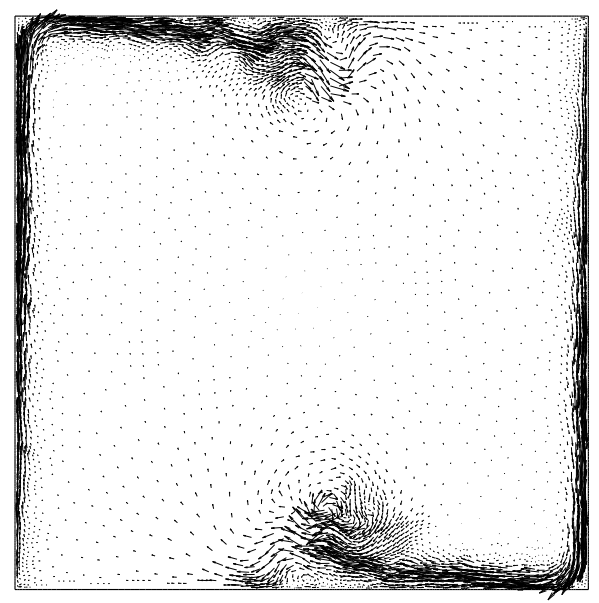

(e) Time step 22, velocity vectors.

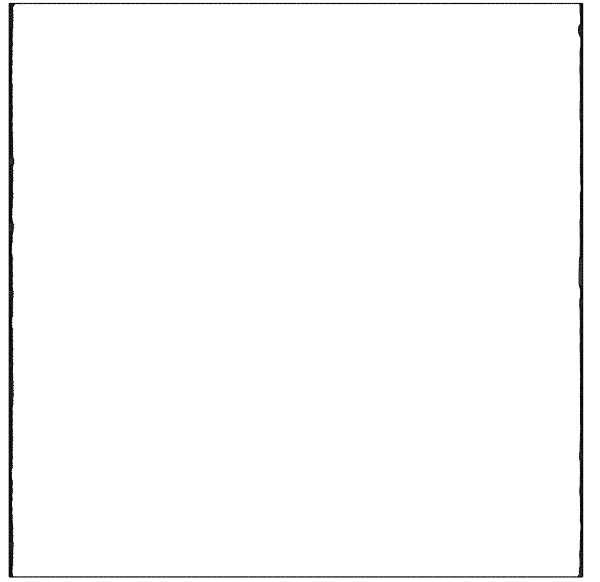

(b) Time step 1, isotherms.

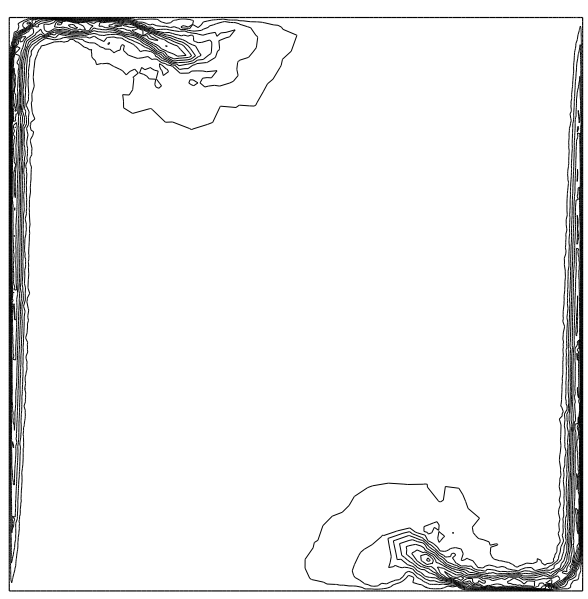

(d) Time step 14, isotherms.

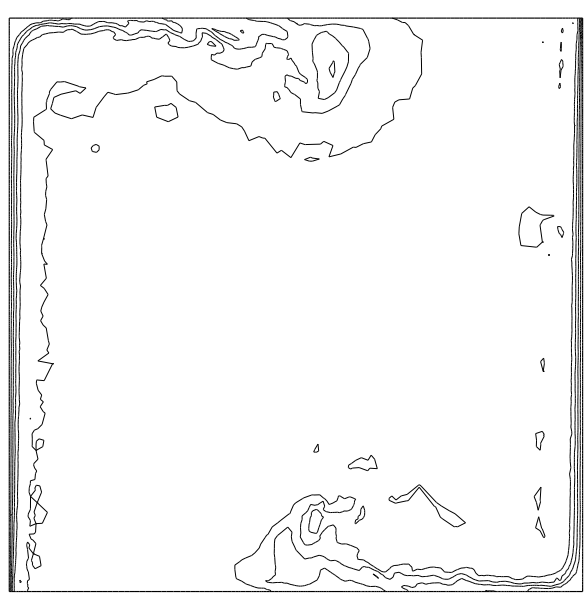

(f) Time step 22, isotherms.

Figure 5: Velocity vectors and isotherms for $\mathrm{Ra}=4 \times 10^{8}$ at (a),(b) $t^{*}=0.0$; (c),(d) $t^{*}=0.00028 ;(\mathrm{e}),(\mathrm{f}) t^{*}=0.00044$. 


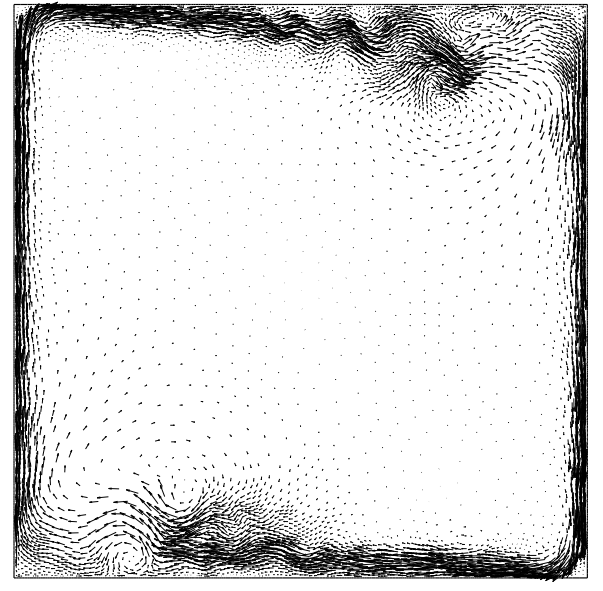

(a) Time step 33, velocity vectors.

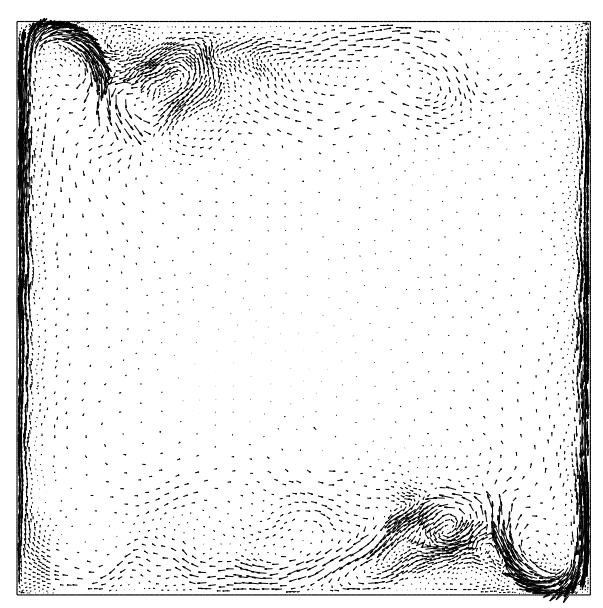

(c) Time step 69, velocity vectors.

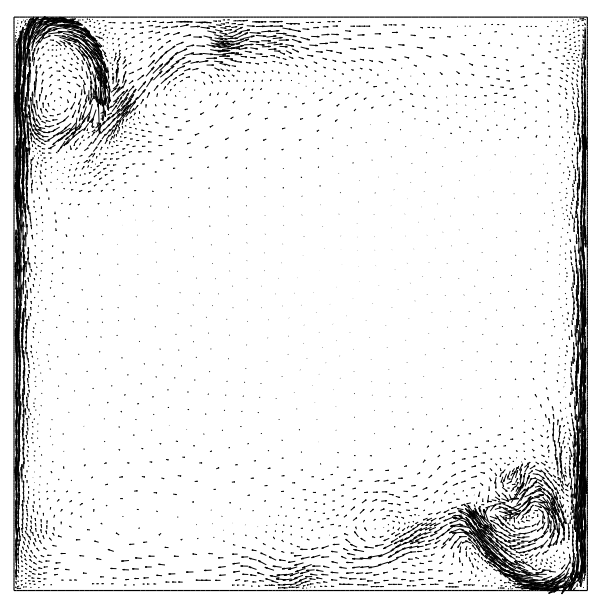

(e) Time step 78, velocity vectors.

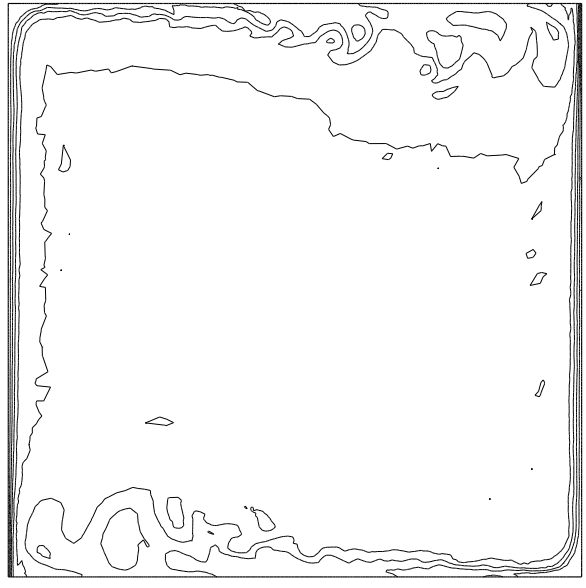

(b) Time step 33, isotherms.

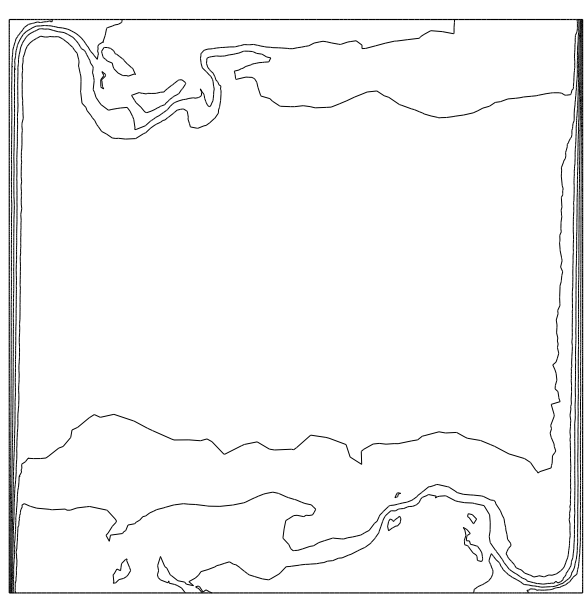

(d) Time step 69, isotherms.

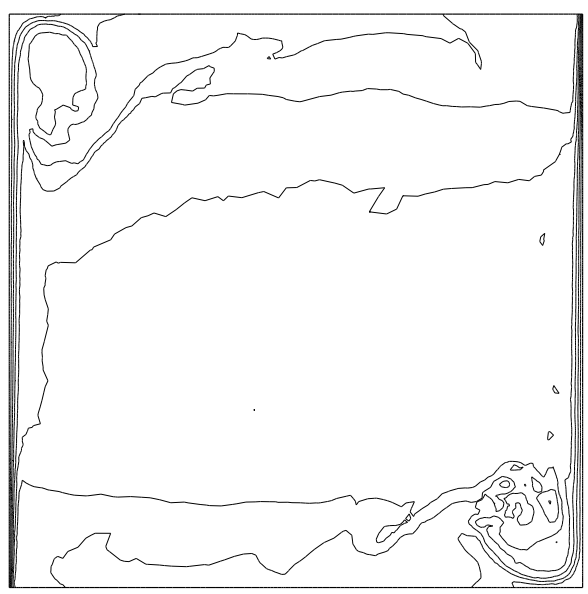

(f) Time step 78, isotherms

Figure 6: Velocity vectors and isotherms for $\mathrm{Ra}=4 \times 10^{8}$ at (a),(b) $t^{*}=0.00066$; (c),(d) $t^{*}=0.00138 ;(\mathrm{e})$,(f) $t^{*}=0.00156$. 


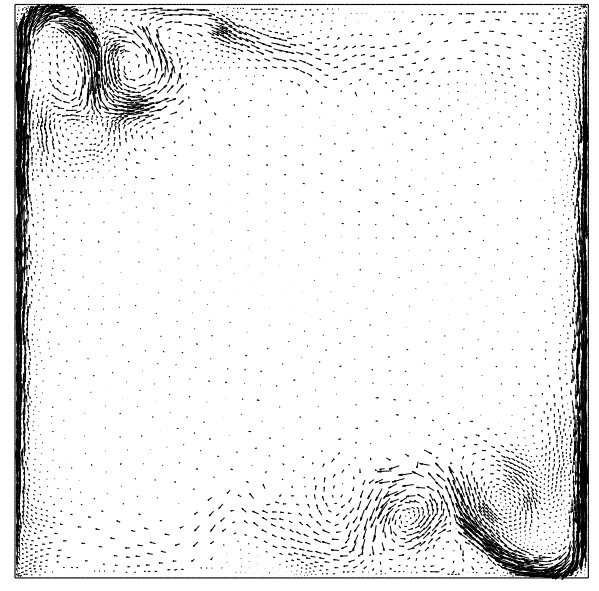

(a) Time step 188, velocity vectors.

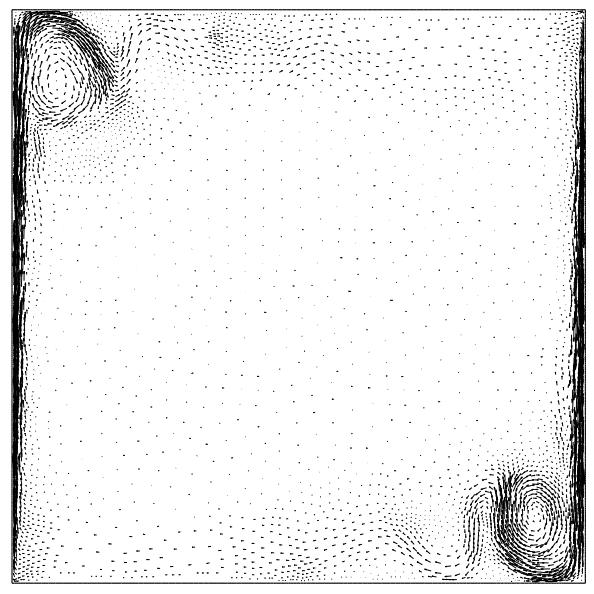

(c) Time step 468, velocity vectors.

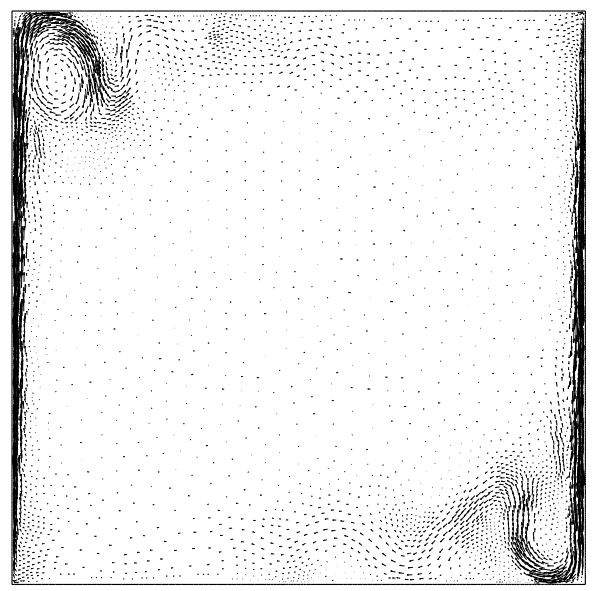

(e) Time step 748, velocity vectors.

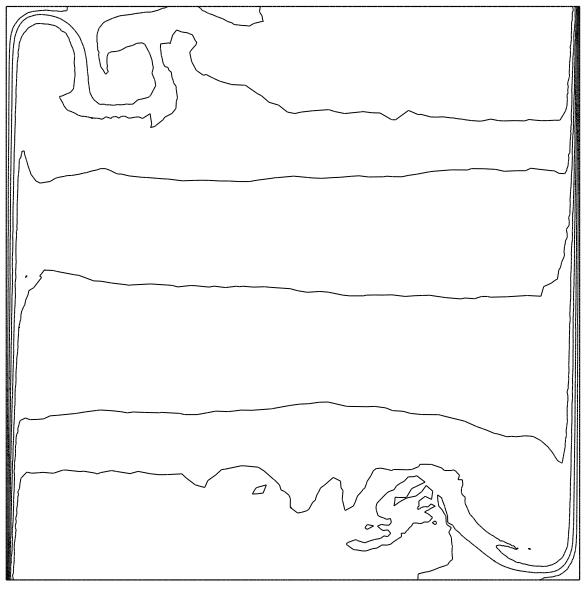

(b) Time step 188, isotherms.

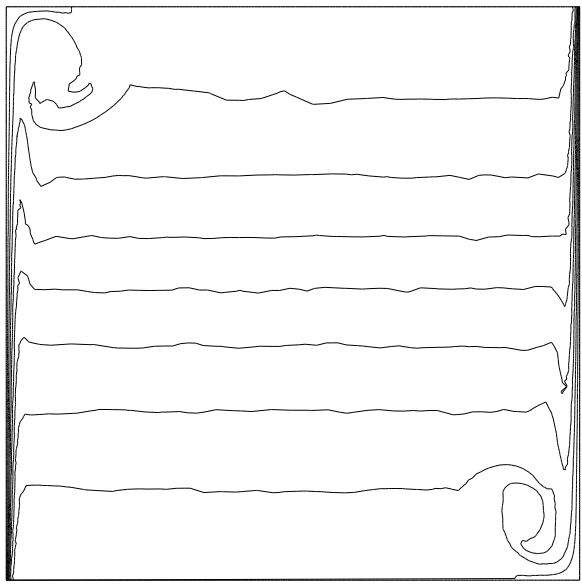

(d) Time step 468, isotherms.

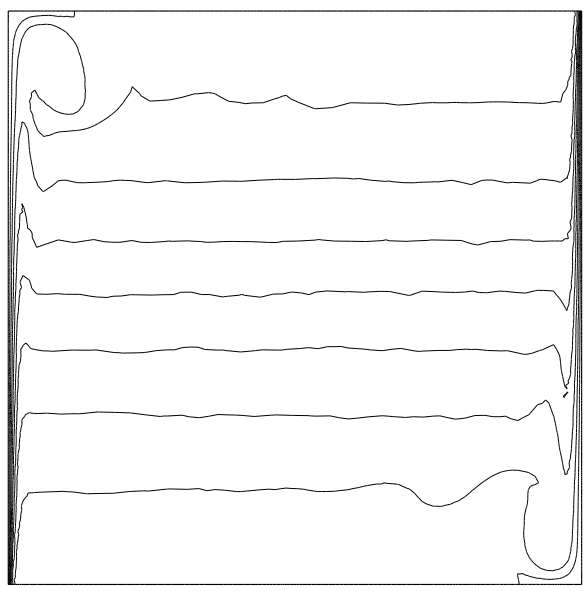

(f) Time step 748, isotherms

Figure 7: Velocity vectors and isotherms for $\mathrm{Ra}=4 \times 10^{8}$ at (a),(b) $t^{*}=0.00376$; (c),(d) $t^{*}=0.01808 ;(\mathrm{e}),(\mathrm{f}) t^{*}=0.03488$. 


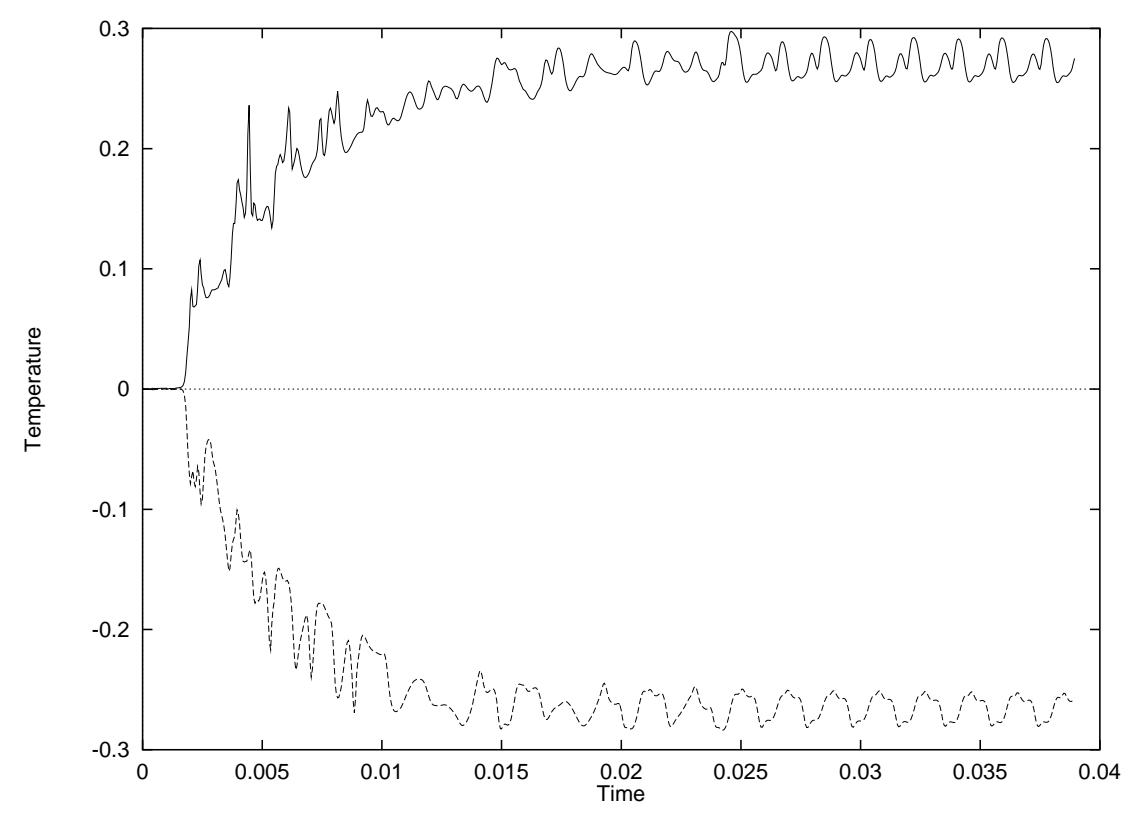

Figure 8: Temperature time trace for $\mathrm{Ra}=2 \times 10^{8}$

\section{Conclusions}

It was demonstrated that $h$-adaptivity with GFEM provides a powerful means of solving difficult problems such as the thermally driven cavity problem at high Rayleigh numbers characterised by; thin boundary layers, separation and recirculation zones and oscillatory internal waves dominating the flow behaviour. The use of $h$-adaptivity produces an accurate, efficient and economical solution to this problem. The accuracy compared favourably with other published solutions. $h$-adaptive methods with automatic mesh refinement based upon the actual physics of the problem are inherently efficient as no development time is required to create the 'right' mesh for a problem. They are also economical as an 'optimal' discretisation is produced for a desired level of accuracy, with grid-points placed only where they are needed. The actual computational time is divided between the solution of the discretised governing equations and the adaptive process (gradient recovery, error-estimation and mesh refinement). The adaptive process accounts for only $0.25 \%$ of the total CPU time. This can be reduced considerably by using simpler structured meshes with a mesh enrichment method of refinement.

It is clear that this problem is dominated by the advective transport mechanism, however the solutions achieved do not rely upon any special scheme for advection dominated flow, such as SUPG etc. This is a very significant additional benefit of using adaptivity in the context of transient problems (especially when a pre-adaptive cycle is performed on the initial conditions). This was alluded to by an early paper by Gresho et al [3] and recently demonstrated by Usmani [1].

Fundamental frequencies were calculated for three Rayleigh numbers; $2 \times 10^{8}, 3 \times 10^{8}$ and $4 \times 10^{8}$. These frequencies were found to be slightly lower than previously calculated 


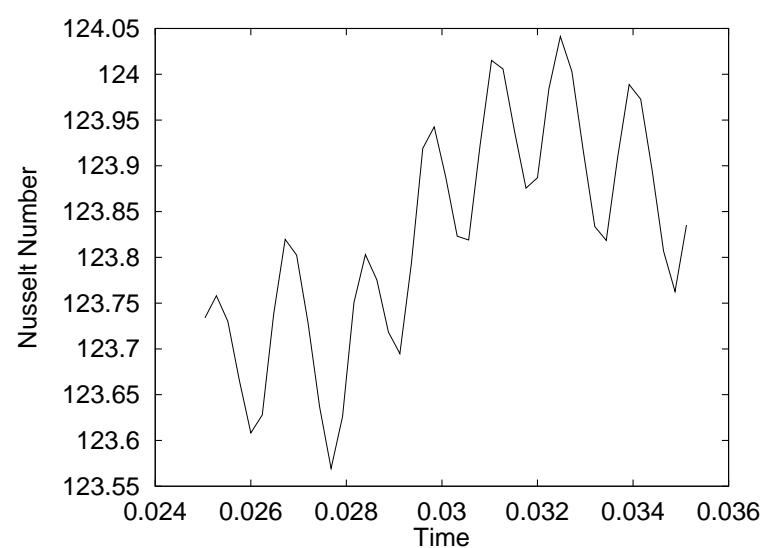

(a) Maximum Nusselt number

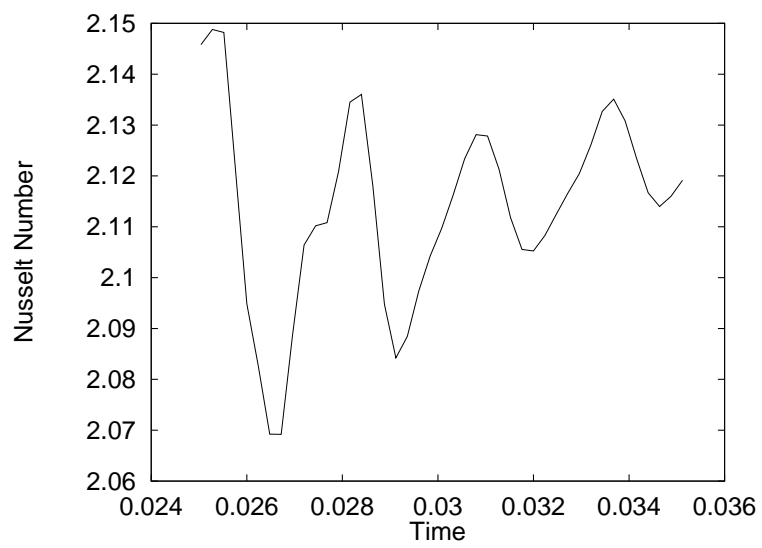

(b) Minimum Nusselt number

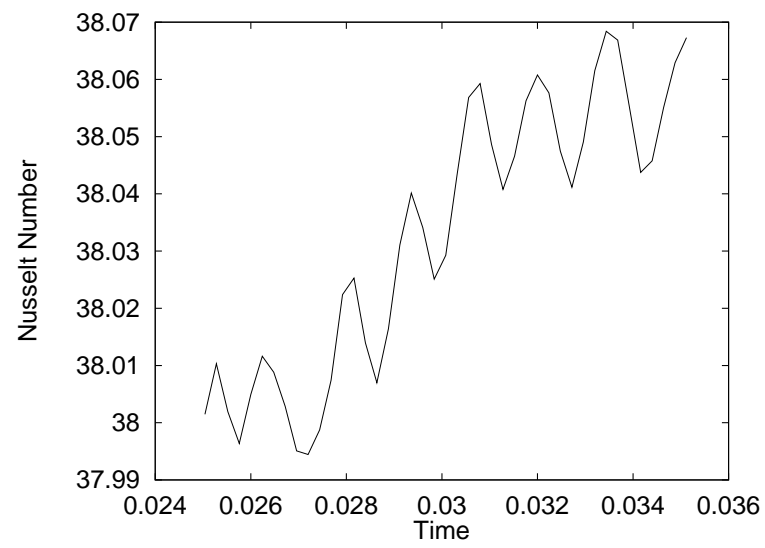

(c) Average Nusselt number

Figure 9: Time plots of Nusselt number on $x=0.0$ for $\mathrm{Ra}=2 \times 10^{8}$ 


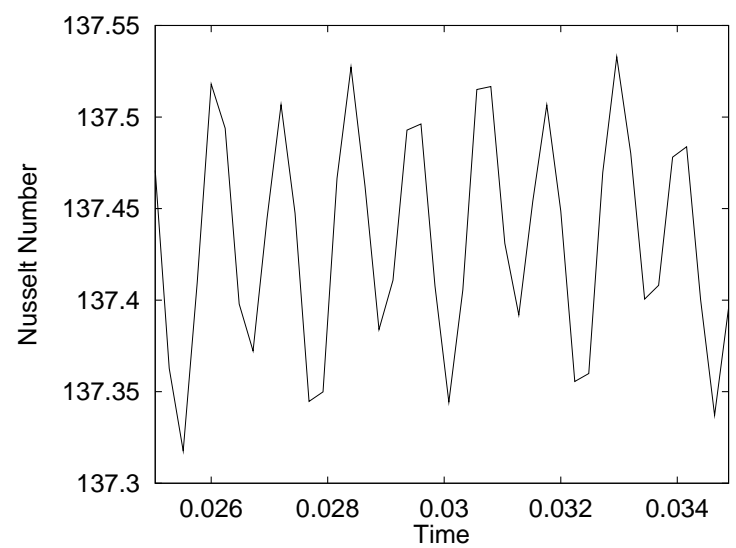

(a) Maximum Nusselt number

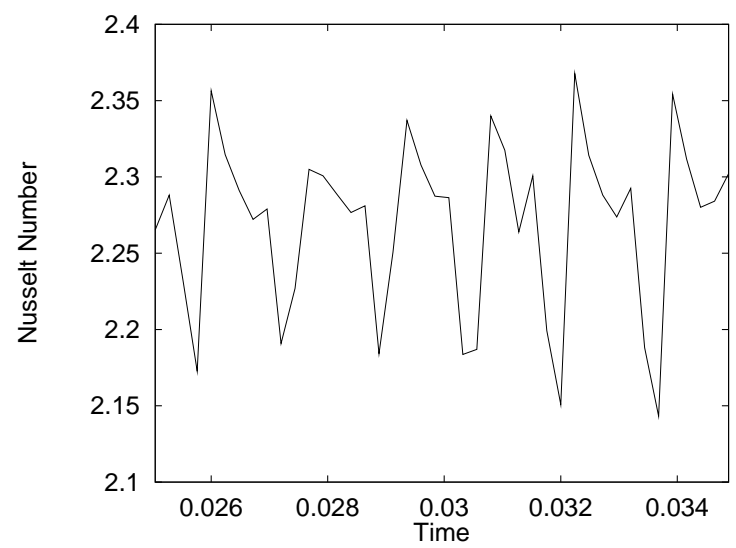

(b) Minimum Nusselt number

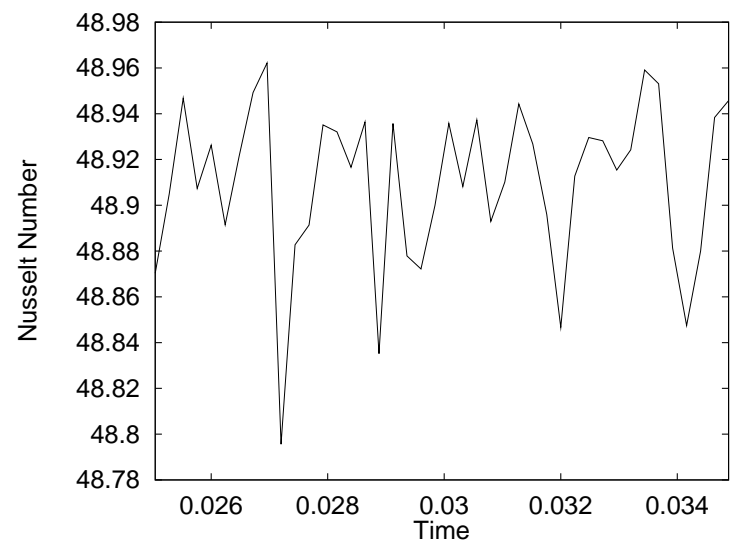

(c) Average Nusselt number

Figure 10: Time plots of Nusselt number on $x=0.0$ for $\mathrm{Ra}=3 \times 10^{8}$ 


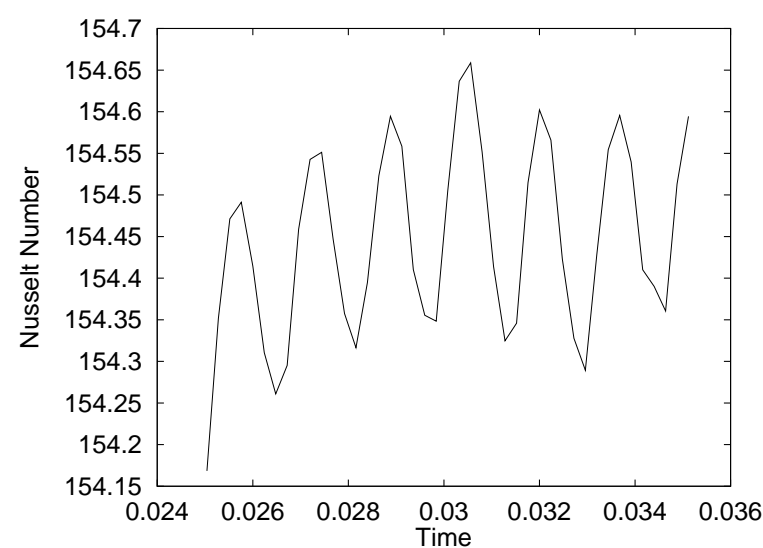

(a) Maximum Nusselt number

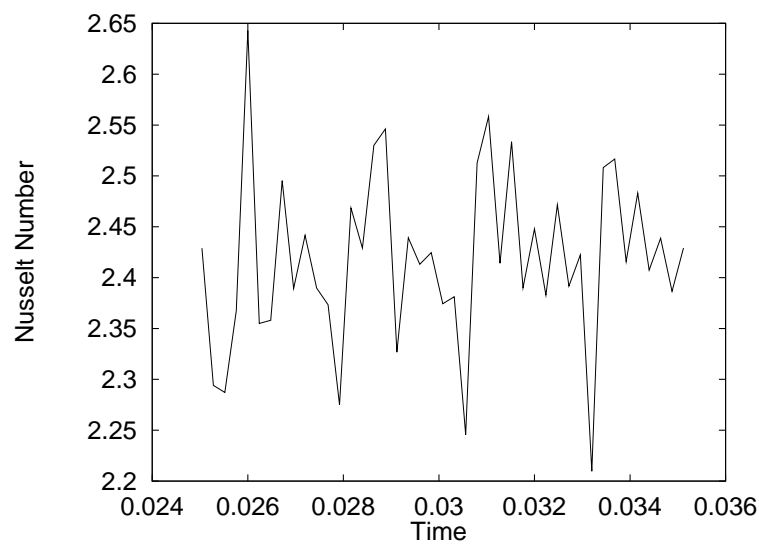

(b) Minimum Nusselt number

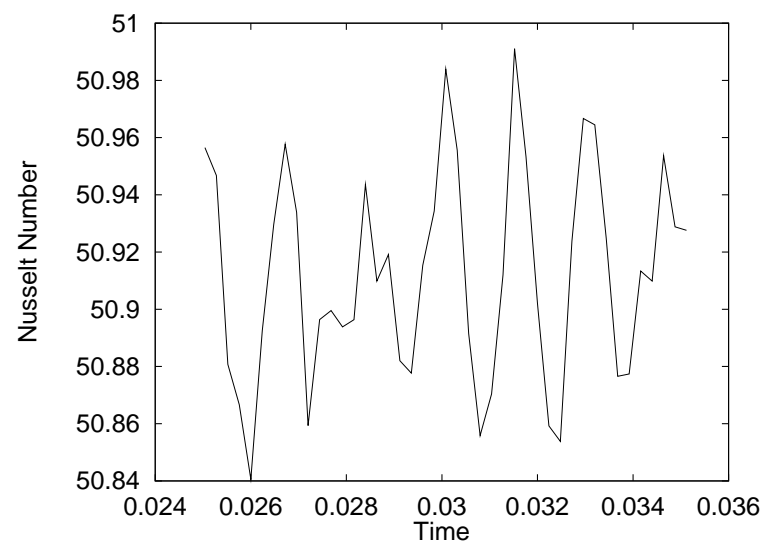

(c) Average Nusselt number

Figure 11: Time plots of Nusselt number on $x=0.0$ for $\mathrm{Ra}=4 \times 10^{8}$ 
by [10]. The primitive variable time history results indicate that the transition from periodic to quasi-periodic and quasi-periodic to mildy chaotic flow match those compiled from past results. Further details such as the possible phase differences between the oscillations at the two departing corners could not be investigated here as an unstructured mesh generator was used in this work. Unless one is prepared to refine to a much lower mesh size (which will be very expensive) it is difficult to separate the effects caused by small differences in the local mesh refinement and genuine flow features. A structured mesh version of this program is under development, which will allow such investigations to be undertaken reliably.

\section{Acknowledgements}

This work was funded by an EPSRC research studentship. The authors are also grateful to Dr M.O.Gordon for his assistance with the FFT analysis. 


\section{References}

[1] A.S.Usmani. Solution of steady and transient advection problems using an h-adaptive finite element method. International Journal of Computational Fluid Dynamics, 11:249-259, 1999.

[2] D.A.Mayne, A.S.Usmani, and M.Crapper. h-adaptive finite element solution of high rayleigh number thermally driven cavity problem. International Journal of Numerical Methods in Heat and Fluid flow, 10(6):598-615, 2000.

[3] P.M. Gresho and R.L.Lee. Don't suppress the wiggles - they're telling you something! The American Society of Mechanical Engineers - Applied Mechanics Division, 34:3761, 1979.

[4] A.N.Brooks and T.J.R.Hughes. Streamline upwind/petrov-galerkin formulations for convection dominated flows with particular emphasis on the incompressible navierstokes equations. Computer Methods in Applied Mechanics and Engineering, 32:199$259,1982$.

[5] G. De Vahl Davis. Natural convection in a square cavity: A comparison exercise. In Second International Conference for Numerical Methods in Thermal Problems, pages 552-572, Venice,Italy, July 1981.

[6] R.F.Bergholz. Instability of steady natural convection in a vertical fluid layer. Journal of Fluid Mechanics, 84:743-768, 1978.

[7] J.Patterson and J.Imberger. Unsteady natural convection in a rectangular cavity. Journal of Fluid Mechanics, 100:65-86, 1980.

[8] M.R.Ravi, R.A.W.M.Henkes, and C.J.Hoogendoorn. On the high rayleigh number structure of steady laminar natural convection flow in a square enclosure. Journal of Fluid Mechanics, 262:325-351, 1994.

[9] G.N.Ivey. Experiments on transient natural convection in a cavity. Journal of Fluid Mechanics, 144:389-401, 1984.

[10] D.R.Chenoweth and S.Paolucci. Natural convection in an enclosed vertical air layer with large horizontal temperature differences. Journal of Fluid Mechanics, 169:173$210,1986$.

[11] S.Paolucci and D.R.Chenoweth. Transition to chaos in a differentially heated vertical cavity. Journal of Fluid Mechanics, 201:379-410, 1989.

[12] P.Haldenwang. Unsteady numerical simulation by chebyshev spectral methods of natural convection at high rayleigh number. American Society of Mechanical Engineering, Heat Transfer Division, 60:45-51, 1986.

[13] P.Haldenwang and G.Labrosse. 2-d and 3-d spectral chebyshev solutions for free convection at high rayleigh number. In Finite Element Methods in Flow Problems, pages 261-266, 1986. 
[14] S.W.Armfield and J.C.Patterson. Direct simulation of wave interactions in unsteady natural convection in a cavity. International Journal for Heat and Mass Transfer, 34:929-940, 1991.

[15] P.M.Gresho, R.L.Lee, S.T.Chan, and R.L.Sani. Solution of the time-dependent incompressible navier-stokes and boussinesq equations using the galerkin finite element method. In Proceedings of the IUTAM Symposium on Approximate Methods for Navier-Stokes Problems, pages 203-222, Paderborn,West Germany, September 1979.

[16] P.M.Gresho, R.L.Lee, and R.L.Sani. On the time-dependent solution of the incompressible Navier-Stokes equations in two and three dimensions. In Recent Advances in Numerical Methods in Fluids, volume 1. Pineridge Press Limited, Swansea, 1980.

[17] A.S.Usmani, R.W.Lewis, and K.N.Seetharamu. Finite element modelling of naturalconvection-controlled change of phase. International Journal for Numerical Methods in Fluids, 14:1019-1036, 1992.

[18] T.J.R.Hughes. Analysis of transient algorithms with particular reference to stability behaviour. In Computational Methods for Transient Analysis. Elsevier Science Publishers, 1983.

[19] T.J.R.Hughes. The Finite Element Method - Linear Static and Dynamic Finite Element Analysis. Prentice-Hall International, Inc., New Jersey, 1987.

[20] H.C.Huang and A.S.Usmani. The Finite Element Analysis for Heat Transfer. Springer-Verlag, 1994.

[21] O.C.Zienkiewicz and J.Z.Zhu. Adaptivity and mesh generation. International Journal for Numerical Methods in Engineering, 32:783-810, 1991.

[22] E.Hinton and J.S.Campbell. Local and global smoothing of discontinuous finite element functions using a least squares method. International Journal for Numerical Methods in Engineering, 8:461-480, 1974.

[23] R.W.Lewis, H.C.Huang, A.S.Usmani, and J.T.Cross. Finite element analysis of heat transfer and flow problems using adaptive remeshing including application to solidification problems. International Journal for Numerical Methods in Engineering, 32:767-781, 1991.

[24] P.Le Quere. Accurate solutions to the square thermally driven cavity at high rayleigh number. Computers and Fluids, 20:29-41, 1991.

[25] P.Le Quere and T. Alziary De Roquefort. Transition to unsteady natural convection of air in differentially heated cavities. American Society of Mechanical Engineering, Heat Transfer Division, 60:29-36, 1986. 